\title{
Equilibration dynamics in nuclear reactions
}

\author{
A.S. Umar ${ }^{1, \star}$, C. Simene ${ }^{2, \star \star}$, and S. Ayik ${ }^{3, \star \star \star}$ K. Godbey ${ }^{1, \star \star \star \star}$ \\ ${ }^{1}$ Department of Physics and Astronomy, Vanderbilt University, Nashville, TN 37235, USA \\ ${ }^{2}$ Department of Theoretical Physics and Department of Nuclear Physics, Research School of Physics and Engineering, The \\ Australian National University, Canberra ACT 2601, Australia \\ ${ }^{3}$ Physics Department, Tennessee Technological University, Cookeville, TN 38505, USA
}

\begin{abstract}
We discuss the equilibration dynamics and time-scales for various quantities that are connected to the experimentally observable entities. These include the study of mass, isospin, and total kinetic energy (TKE) equilibration time-scales as well as the time-scale for fluctuations.
\end{abstract}

\section{Introduction}

Heavy-ion reactions at low energies carry a wealth of information to investigate the characteristic features of nuclear dynamics. Furthermore, these reactions help explore the intriguing interplay between quantal dynamics at the microscopic level and the collective dynamics on timescales that are too short for full equilibration. To fully classify the systematics of these reactions both theoretical and experimental studies are required for a diverse selection of systems. With the development of radioactive ion-beam facilities this goal is rapidly evolving.

In this proceeding we provide a discussion and microscopic calculations to examine the equilibration dynamics and time-scales for various quantities that are connected to the experimentally observable entities. These include the study of mass, isospin, and total kinetic energy (TKE) equilibration time-scales as well as the time-scale for fluctuations. The phenomena discussed here is directly related to the transport dynamics between reacting systems that initially carry a quantal asymmetry. A recent example of such a study is the experiment to investigate isospin equilibration in the vicinity of the Fermi energy $[1,2]$. Earlier studies at lower energies involved deep-inelastic reaction of systems with large isospin asymmetry [3-5].

Below, we utilize the time-dependent Hartree-Fock (TDHF) theory [6] to investigate a selected number of systems. A more comprehensive study involving many more systems will be the subject of a future publication.

\section{Equilibration from TDHF}

Low-energy nuclear reactions encompass very diverse time-scales depending on the type of collision studied.

\footnotetext{
^e-mail: umar@compsci.cas.vanderbilt.edu

$\star \star$ e-mail: cedric.simenel@anu.edu.au

$\star \star \star$ e-mail: Ayik@tntech.edu

$\star \star \star \star$ e-mail: kylegodbey@gmail.com
}

At times immediately after contact $\left(\sim 10^{-22}\right.$ sec. $)$ preequilibrium particle emission can take place. This is followed by the deep-inelastic regime (a few times $10^{-21} \mathrm{sec}$.) and the fast quasifission regime $\left(\sim 10^{-21}-10^{-20} \mathrm{sec}\right.$.). For these longer sticking times the outgoing channel begins to loose the memory of the entrance channel properties. Yet at longer time-scales the system becomes partially or fully equilibrated leading to slow quasifission followed by fusion-fission, and finally compound nucleus formation or compound nucleus fission. The latter processes are relatively well understood in the context of models relying on statistical equilibrium physics. The challenging aspect is the intermediary regime, where the dynamics and shell effects still play a role and equilibration of various quantities occur at different time-scales. Among these here we study the equilibration of mass, isospin, kinetic energy, and fluctuations. For numerical calculations we employ a fully 3D TDHF code [7-9] and the SLy4d [10] Skyrme energy density functional. The plotted results are comprised of calculations involving many partial waves and therefor show large fluctuations.

\subsection{Mass}

Quasifission provides a unique probe to quantum manybody dynamics of out-of-equilibrium nuclear systems. For instance, quasifission studies bring information on mass equilibration time-scales [11-13], on shell effects in the exit channels [14-18], dependence on the neutron-toproton ratio $N / Z$ of the compound nucleus [19], as well as on the nuclear equation of state [20,21]. The TDHF theory has been employed to study quasifission dynamics with considerable success [22-29]. The outcome of the calculations strongly depend on the orientation of the deformed nuclei. Here, we discuss the ${ }^{48} \mathrm{Ca}+{ }^{249} \mathrm{Bk}$ reaction with the TDHF approach. Unlike previous calculations where the TDHF studies of quasifission with actinide targets were restricted to one or two orientations, we performed calcu- 
lations spanning both a range of orientations and a range of angular momenta. The orientation of the deformed ${ }^{249} \mathrm{Bk}$ was changed by $15^{\circ}$ steps to cover the full range $(0, \pi)$ with orbital angular momentum $L$ changing in units of $10 \hbar$ from 0 to quasielastic collisions.

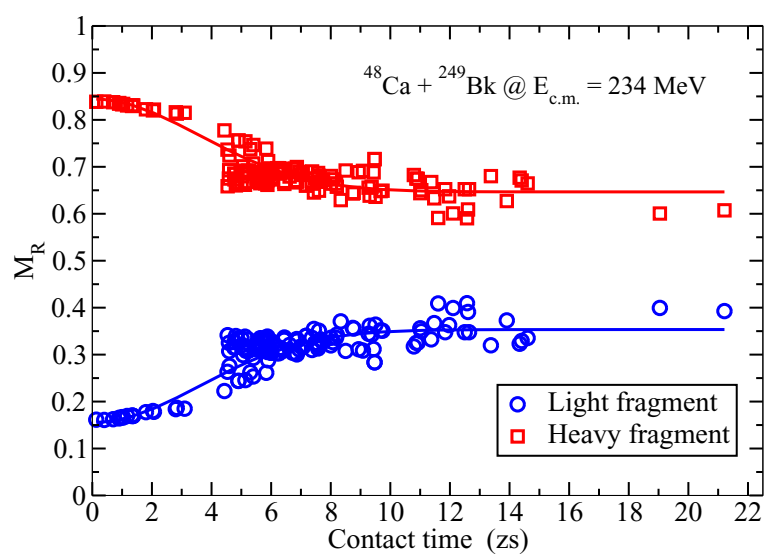

Figure 1. For the ${ }^{48} \mathrm{Ca}+{ }^{249} \mathrm{Bk}$ reaction; Mass ratio of fragment masses as a function of contact time at $E_{\mathrm{c} . \mathrm{m} .}=234 \mathrm{MeV}$. The solid lines show possible fits.

Figure 1 shows the mass ratio, $M_{R}=M_{\text {frag }} / M_{\text {total }}$, of fragment masses as a function of contact time at $E_{\text {c.m. }}=$ $234 \mathrm{MeV}$ for the ${ }^{48} \mathrm{Ca}+{ }^{249} \mathrm{Bk}$ reaction. We define the contact time as the time interval between the time $t_{1}$ when the two nuclear surfaces (defined as isodensities with half the saturation density $\rho_{0} / 2=0.07 \mathrm{fm}^{-3}$ ) first merge into a single surface and the time $t_{2}$ when the surface splits up again. The solid lines are a simple fit to the results. We observe

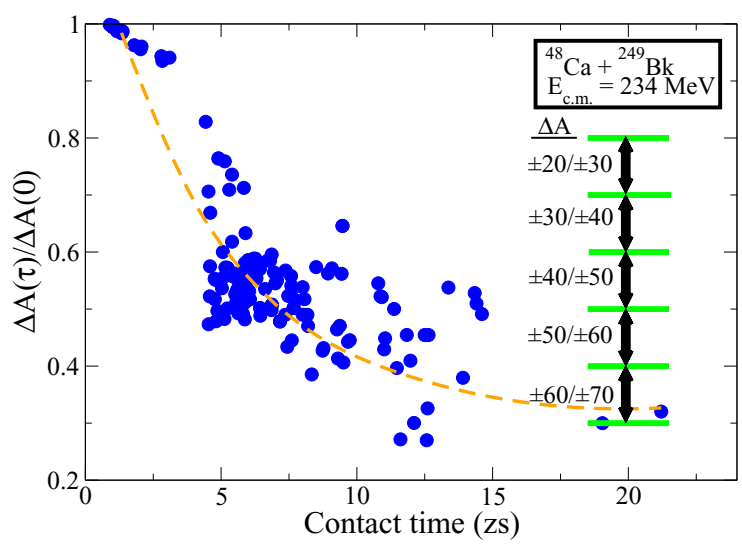

Figure 2. The ratio of the final and initial fragment masses as a function of contact time for the ${ }^{48} \mathrm{Ca}+{ }^{249} \mathrm{Bk}$ system at $E_{\text {c.m. }}=$ $234 \mathrm{MeV}$ and for all orientations and orbital angular momenta discussed in the text. The horizontal bars on the left indicate the intervals for the net number of transferred particles. The dashed line shows one possible fit.

that mass equilibration appears to occur for times larger than about $8-10 \mathrm{zs}$, starting at about $6 \mathrm{zs}$. Another perspective of these results can be obtained by plotting the ratio of the final fragment mass difference to the initial mass difference as a function of contact time, as shown in Fig. 2 for the same system. The horizontal lines on the right indi- cate the intervals for the net number of transferred particles and the dashed line is a possible fit to guide the eye.

From these results and a number of others not shown here we can conclude that the mass equilibration is substantially longer as expected in comparison to the other quantities mentioned below. It is also interesting to observe that there is a bunching of results around certain mass ratios. This is shown to be related to shell effects influencing the dynamical quasifission process in Ref. [30].

\subsection{Isospin and TKE}

Transport properties of isospin asymmetric nuclear matter can be investigated by studying charge equilibration driven by the nuclear symmetry-energy in heavy-ion collisions. For example, collisions at Fermi energies give access to contact times which are short enough to induce only a partial charge equilibration [31] and thus can be used to determine equilibration times [1]. Due to a faster time scale for equilibration of charge and total kinetic energy (TKE), deep-inelastic collisions are the preferred venue at lower energies. We have done a systematic study of the ${ }^{78,92} \mathrm{Kr}+{ }^{208} \mathrm{~Pb}$ systems at $8.5 \mathrm{MeV} /$ nucleon. In Fig. 3 we

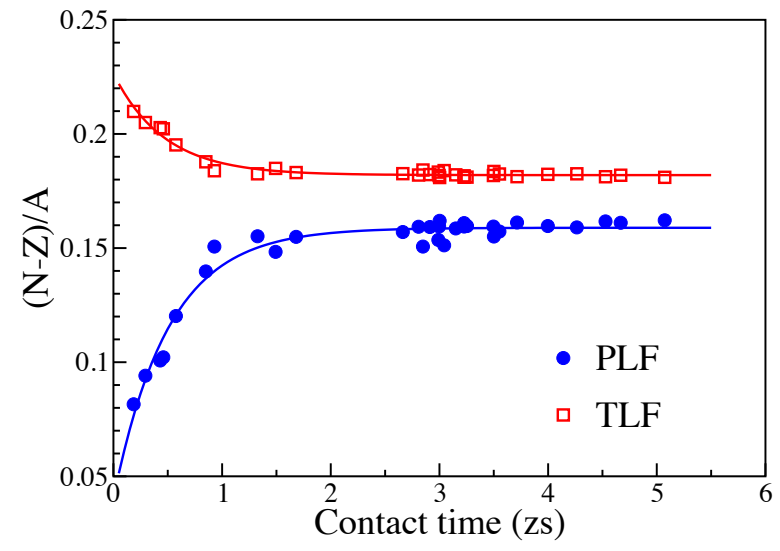

Figure 3. The $(N-Z) / A$ value of the primary light (full circles) and heavy (open squares) fragments formed in ${ }^{78} \mathrm{Kr}+{ }^{208} \mathrm{~Pb}$ at $E=8.5 \mathrm{MeV} /$ nucleon are plotted as a function of the contact time between the collision partners. The solid lines show fits to the TDHF results.

plot the ratio $(N-Z) / A$ as a function of contact time for the ${ }^{78} \mathrm{Kr}+{ }^{208} \mathrm{~Pb}$ system. An equilibration time $\tau \sim 0.5 \mathrm{zs}$ is obtained from the fit $(N-Z) / A=\alpha+\beta \exp (-T / \tau)$ to the TDHF results as it was done in Ref. [1]. This is in agreement with a slightly faster equilibration time obtained $(\sim 0.3 \mathrm{zs})$ for reactions near the Fermi energy [1].

In order to study the equilibration of TKE in Fig. 4 we plot the TKE of the final fragments as a function of contact time for ${ }^{78,92} \mathrm{Kr}+{ }^{208} \mathrm{~Pb}$ systems. The two orientations of the deformed $\mathrm{Kr}$ nuclei are also indicated on the plot. We observe that the equilibration of the TKE is also a relatively fast process but somewhat longer than that of isospin transfer. The TKE seems to reach its equilibrium value at around $1.5 \mathrm{zs}$. 


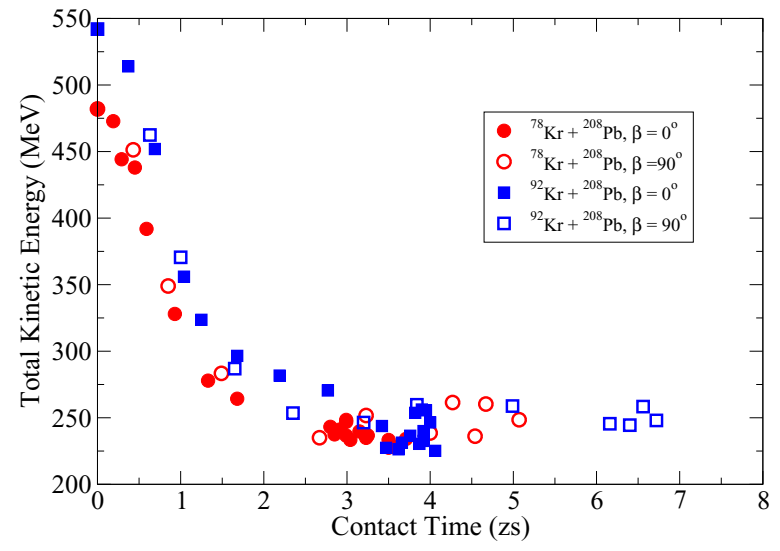

Figure 4. The exit channel TKE in ${ }^{78} \mathrm{Kr}+{ }^{208} \mathrm{~Pb}$ reaction at $E=$ $8.5 \mathrm{MeV} /$ nucleon are plotted as a function of the contact time between the collision partners.

\subsection{Fluctuations}

The mean-field description of reactions using TDHF provides the mean values of the proton and neutron drift. It is also possible to compute the probability to form a fragment with a given number of nucleons [32-35], but the resulting fragment mass and charge distributions are often underestimated in dissipative collisions [36,37]. Much effort has been done to improve the standard mean-field approximation by incorporating the fluctuation mechanism into the description. At low energies, the mean-field fluctuations make the dominant contribution to the fluctuation mechanism of the collective motion. Various extensions have been developed to study the fluctuations of onebody observables. These include the TDRPA approach of Balian and Vénéroni [38], the time-dependent generator coordinate method [39], or the stochastic mean-field (SMF) method [40,41]. In the SMF approach the collision dynamics is described in terms of an ensemble of mean-field events. The single-particle density matrix of each event, $\rho^{\lambda}(\vec{r}, \vec{p}, t)$, is determined by the self-consistent mean-field of the corresponding event. Here $\lambda$ indicates the event label. When a di-nuclear structure is maintained during the collision, such as deep inelastic and quasifission reactions, we do not need to generate such an ensemble of mean-field events. In this case it is possible to describe the collision dynamics in terms of a few relevant macroscopic variables, such as mass and charge asymmetry, the relative linear momentum and the orbital angular momentum. In di-nuclear geometry, we can express these macroscopic variables in terms of the TDHF solutions with the help of window dynamics [42].

In Fig. 5a we plot the time-evolution of the calculated SMF co-variances for the collision of ${ }^{60} \mathrm{Ni}+{ }^{60} \mathrm{Ni}$, and in In Fig. $5 b$ the same quantities for ${ }^{58} \mathrm{Ni}+{ }^{60} \mathrm{Ni}$, at center of mass energy $E_{\mathrm{c} . \mathrm{m} .}=135.6 \mathrm{MeV}$ and initial orbital angular momentum $L=73 \hbar$. As we see all of the co-variances stabilize around $800-1000 \mathrm{fm} / c$, which corresponds to approximately $2.6-3.3 \mathrm{zs}$.

This work has been supported by the U.S. Department of Energy grant Nos. DE-SC0013847 and DE-SC0015513,

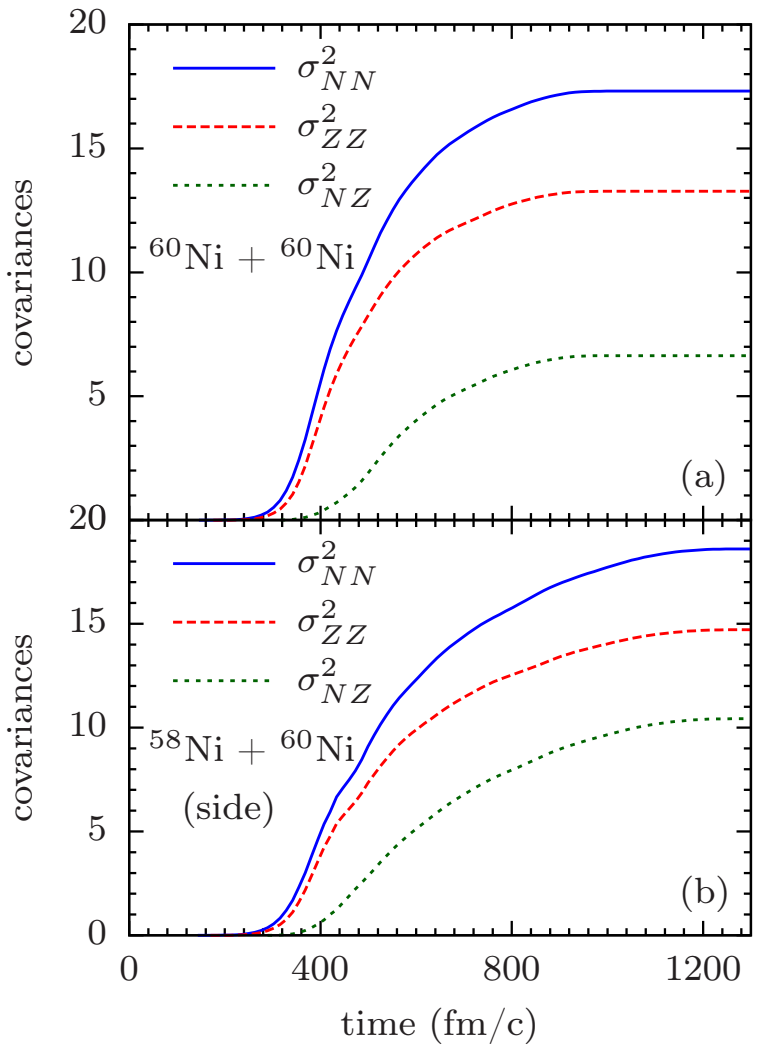

Figure 5. Co-variances in the collision of ${ }^{60} \mathrm{Ni}+{ }^{60} \mathrm{Ni}$ (a) and in the collision of ${ }^{58} \mathrm{Ni}+{ }^{60} \mathrm{Ni}$ in the side geometry (b) at the center of mass energy $E_{\mathrm{c} . \mathrm{m} .}=135.6 \mathrm{MeV}$ and initial orbital angular momentum $L=73 \hbar$.

and by the Australian Research Council Discovery Project (project numbers DP160101254 and DP190100256) funding schemes.

\section{References}

[1] A. Jedele, A.B. McIntosh, K. Hagel, M. Huang, L. Heilborn, Z. Kohley, L.W. May, E. McCleskey, M. Youngs, A. Zarrella et al., Phys. Rev. Lett. 118, 062501 (2017)

[2] Alan B. McIntosh, Sherry J. Yennello, Prog. Part. Nucl. Phys. 108, 103707 (2019)

[3] R. Planeta, S.H. Zhou, K. Kwiatkowski, W.G. Wilson, V.E. Viola, H. Breuer, D. Benton, F. Khazaie, R.J. McDonald, A.C. Mignerey et al., Phys. Rev. C 38, 195 (1988)

[4] R.T. de Souza, W.U. Schröder, J.R. Huizenga, R. Planeta, K. Kwiatkowski, V.E. Viola, H. Breuer, Phys. Rev. C 37, 1783 (1988)

[5] R. Płaneta, K. Kwiatkowski, S.H. Zhou, V.E. Viola, H. Breuer, M.A. McMahan, W. Kehoe, A.C. Mignerey, Phys. Rev. C 41, 942 (1990)

[6] C. Simenel, A.S. Umar, Prog. Part. Nucl. Phys. 103, 19 (2018)

[7] A.S. Umar, M.R. Strayer, J.S. Wu, D.J. Dean, M.C. Güçlü, Phys. Rev. C 44, 2512 (1991)

[8] A.S. Umar, V.E. Oberacker, Phys. Rev. C 73, 054607 (2006) 
[9] A.S. Umar, V.E. Oberacker, Phys. Rev. C 74, 061601 (2006)

[10] Ka-Hae Kim, Takaharu Otsuka, Paul Bonche, J. Phys. G 23, 1267 (1997)

[11] J. Tõke, R. Bock, G.X. Dai, A. Gobbi, S. Gralla, K.D. Hildenbrand, J. Kuzminski, W.F.J. Müller, A. Olmi, H. Stelzer et al., Nucl. Phys. A 440, 327 (1985)

[12] W.Q. Shen, J. Albinski, A. Gobbi, S. Gralla, K.D. Hildenbrand, N. Herrmann, J. Kuzminski, W.F.J. Müller, H. Stelzer, J. Tõke et al., Phys. Rev. C 36, 115 (1987)

[13] R. du Rietz, D.J. Hinde, M. Dasgupta, R.G. Thomas, L.R. Gasques, M. Evers, N. Lobanov, A. Wakhle, Phys. Rev. Lett. 106, 052701 (2011)

[14] M.G. Itkis, J. Äystö, S. Beghini, A.A. Bogachev, L. Corradi, O. Dorvaux, A. Gadea, G. Giardina, F. Hanappe, I.M. Itkis et al., Nucl. Phys. A 734, 136 (2004)

[15] K. Nishio, H. Ikezoe, S. Mitsuoka, I. Nishinaka, Y. Nagame, Y. Watanabe, T. Ohtsuki, K. Hirose, S. Hofmann, Phys. Rev. C 77, 064607 (2008)

[16] E.M. Kozulin, G.N. Knyazheva, S.N. Dmitriev, I.M. Itkis, M.G. Itkis, T.A. Loktev, K.V. Novikov, A.N. Baranov, W.H. Trzaska, E. Vardaci et al., Phys. Rev. C 89, 014614 (2014)

[17] A. Wakhle, C. Simenel, D.J. Hinde, M. Dasgupta, M. Evers, D.H. Luong, R. du Rietz, E. Williams, Phys. Rev. Lett. 113, 182502 (2014)

[18] M. Morjean, D.J. Hinde, C. Simenel, D.Y. Jeung, M. Airiau, K.J. Cook, M. Dasgupta, A. Drouart, D. Jacquet, S. Kalkal et al., Phys. Rev. Lett. 119, 222502 (2017)

[19] K. Hammerton, Z. Kohley, D.J. Hinde, M. Dasgupta, A. Wakhle, E. Williams, V.E. Oberacker, A.S. Umar, I.P. Carter, K.J. Cook et al., Phys. Rev. C 91, 041602(R) (2015)

[20] M. Veselsky, J. Klimo, Y.G. Ma, G.A. Souliotis, Phys. Rev. C 94, 064608 (2016)

[21] H. Zheng, S. Burrello, M. Colonna, D. Lacroix, G. Scamps, Phys. Rev. C 98, 024622 (2018)
[22] Cédric Golabek, Cédric Simenel, Phys. Rev. Lett. 103, 042701 (2009)

[23] David J. Kedziora, Cédric Simenel, Phys. Rev. C 81, 044613 (2010)

[24] V.E. Oberacker, A.S. Umar, C. Simenel, Phys. Rev. C 90, 054605 (2014)

[25] A.S. Umar, V.E. Oberacker, C. Simenel, Phys. Rev. C 92, 024621 (2015)

[26] A.S. Umar, V.E. Oberacker, C. Simenel, Phys. Rev. C 94, 024605 (2016)

[27] K. Sekizawa, K. Yabana, Phys. Rev. C 93, 054616 (2016)

[28] K. Sekizawa, Phys. Rev. C 96, 041601(R) (2017)

[29] K. Sekizawa, K. Hagino, Phys. Rev. C 99, 051602 (2019)

[30] K. Godbey, A.S. Umar, C. Simenel, Phys. Rev. C 100, 024610 (2019)

[31] M.B. Tsang, T.X. Liu, L. Shi, P. Danielewicz, C.K. Gelbke, X.D. Liu, W.G. Lynch, W.P. Tan, G. Verde, A. Wagner et al., Phys. Rev. Lett. 92, 062701 (2004)

[32] S.E. Koonin, K.T.R. Davies, V. Maruhn-Rezwani, H. Feldmeier, S.J. Krieger, J.W. Negele, Phys. Rev. C 15, 1359 (1977)

[33] C. Simenel, Phys. Rev. Lett. 105, 192701 (2010)

[34] Kazuyuki Sekizawa, Kazuhiro Yabana, Phys. Rev. C 88, 014614 (2013)

[35] G. Scamps, D. Lacroix, Phys. Rev. C 87, 014605 (2013)

[36] C.H. Dasso, T. Dossing, H.C. Pauli, Z. Phys. A 289, 395 (1979)

[37] C. Simenel, Phys. Rev. Lett. 106, 112502 (2011)

[38] R. Balian, M. Vénéroni, Ann. Phys. 216, 351 (1992)

[39] H. Goutte, J.F. Berger, P. Casoli, D. Gogny, Phys. Rev. C 71, 024316 (2005)

[40] S. Ayik, Phys. Lett. B 658, 174 (2008)

[41] S. Ayik, B. Yilmaz, O. Yilmaz, Phys. Rev. C 92, 064615 (2015)

[42] S. Ayik, B. Yilmaz, O. Yilmaz, A.S. Umar, Phys. Rev. C 97, 054618 (2018) 